\title{
Cross talk in wavelength-multiplexed holographic memories
}

\author{
Kevin Curtis
}

Northrop B-2 Division, Pico Rivera, California 90660, and Department of Electrical Engineering, California Institute of Technology, MS 116-81, Pasadena, California 91125

Claire Gu

Department of Electrical Engineering, Pennsylvania State University, University Park, Pennsylvania 16802

\section{Demetri Psaltis}

Department of Electrical Engineering, California Institute of Technology, MS 116-81, Pasadena, California 91125

Received December 22, 1992

\begin{abstract}
The cross talk between wavelength-multiplexed holograms is analyzed. The signal-to-noise ratio (SNR) is calculated for a recording schedule that places the center of each image at the null (in wavelength space) of the adjacent hologram. An asymptotic closed-form expression for the minimum SNR is derived in a general reflection geometry. The reflection geometry with counterpropagating signal and reference beams is shown to have the best SNR.
\end{abstract}

One can record multiple holograms in a material by changing either the reference-beam angle ${ }^{1-3}$ (angle multiplexing) or the recording wavelength ${ }^{1,4,5}$ (wavelength multiplexing). These volume holographic memories have potentially high storage capacity and fast (page-at-a-time) readout. In the past, most research concentrated on angle multiplexing, primarily because convenient frequency-tunable light sources were not available. With the advent of semiconductor and solid-state tunable lasers, there has been renewed interest in wavelength multiplexing. Rakuljic et al. ${ }^{5}$ also

$$
\Delta \epsilon \approx \sum_{m=-M}^{M} R_{m}^{*} S_{m}+\text { c.c. },
$$

where c.c. is the complex-conjugate term. The reference wave is given by

$$
R_{m}=\exp \left(i k_{m} r\right)
$$

where $k_{m}$ refers to the wave vector coming from the reference-point source with wavelength $\lambda_{m}$. In addition, with standard Fourier-optics analysis, ${ }^{8}$ the $m$ th signal beam can be expressed as

$$
S_{m}(r) \approx \exp \left(i \frac{2 \pi}{\lambda_{m}} z\right) \iint \mathrm{d} x_{0} \mathrm{~d} y_{0} f_{m}\left(x_{0}, y_{0}\right) \exp \left[-i \frac{2 \pi}{\lambda_{m} F}\left(x x_{0}+y y_{0}\right)\right] \exp \left[-i \frac{\pi z}{\lambda_{m} F^{2}}\left(x_{0}{ }^{2}+y_{0}^{2}\right)\right]
$$

argued that wavelength-multiplexed memories have low cross-talk noise.

The information storage capacity of volumeholographic memories is limited by geometry and material dynamic range. The signal-to-noise ratio (SNR) is a convenient measure used to assess the storage capability of holographic memories. ${ }^{6,7}$ In this Letter we calculate the SNR that results from cross talk between holograms that are purely due to geometric constraints.

Fourier-transform holograms are wavelength multiplexed in a volume holographic medium with the setup shown in Fig. 1. A plane wave $R_{m}$ is generated at wavelength $\lambda_{m}$ and angle $\theta$ with respect to the $z$ axis by collimating light from a point source. This interferes inside the holographic medium with $S_{m}$, the Fourier-transform of the $m$ th object image, also of wavelength $\lambda_{m}$. When we label the holograms $m=$ $-M,-(M-1), \ldots, 0, \ldots, M$, the presence of these $N=2 M+1$ holograms modulates the permittivity of the material so that the change in the permittivity of the medium can be written as where $f_{m}\left(x_{0}, y_{0}\right)$ is the $m$ th object image; $x, y$, and $z$ are the coordinates at the back focal plane of lens $\mathrm{L}_{1}$; and $F$ is the focal length of all three lenses, $\mathrm{L}_{1}$, $L_{2}$, and $L_{3}$. By substituting relations (2) and (3) into relation (1), one can write $\Delta \epsilon$ explicitly.

The recorded medium is illuminated with one of the reference plane waves. Ideally, this reconstructs the

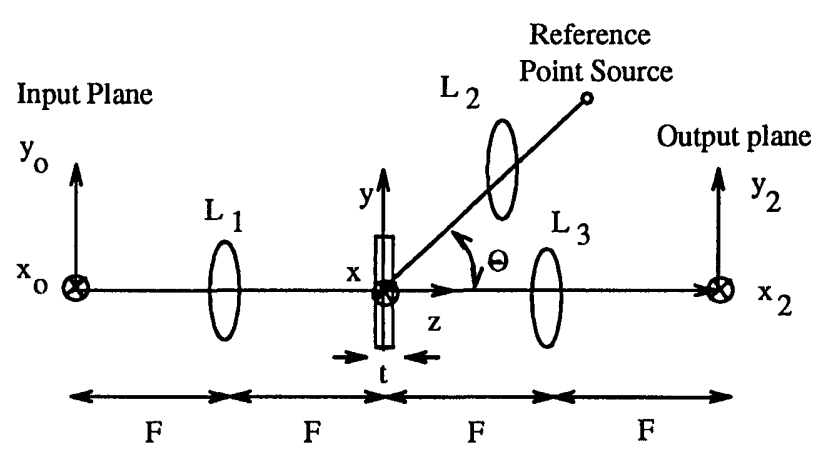

Fig. 1. Recording and readout geometry for wavelength multiplexing. 
hologram associated with that particular reference. In what follows we calculate the deviations of the reconstructed wave from the desired image. The diffracted plane-wave component $E(r)$, with wave vector $k_{d, j}$ and wavelength $\lambda_{j}$, can be derived from standard scalar diffraction theory. ${ }^{9}$ The following expression assumes that the Born and paraxial approximations are valid:

$E(r) \approx \frac{k_{0}^{2}}{4 \pi} \frac{\exp (i k r)}{r} \int \mathrm{d} r^{\prime} \exp \left(-i K r^{\prime}\right) \Delta \epsilon\left(r^{\prime}\right)$,

where $K=k_{d, j}-k_{j}$ and $k_{j}$ is the illuminating reference beam with wavelength $\lambda_{j}$. The stored perturbation $\Delta \epsilon$ contains a grating-vector component $K$ that will give the desired response as a strong contribution to the integral in relation (4). However, the finite volume over which the integration is carried out causes frequency components other than $K$ to also yield nonzero contributions to the above integral. Therefore relation (4) describes both the diffracted light caused by the desired $S_{j}$ and the noise caused by the diffracted light from the other holograms.

Using relations (1)-(4), we can explicitly state the diffracted light resulting from the presence of the permittivity perturbation. This result can then be related to the detected pattern by using the paraxial approximation to describe how lens $\mathrm{L}_{3}$ maps $k_{d, j}$ to a point $x_{2}, y_{2}$ at the output plane:

$k_{d, j}=\left[\frac{2 \pi x_{2}}{\lambda_{j} F}, \frac{2 \pi y_{2}}{\lambda_{j} F}, \frac{2 \pi}{\lambda_{j}}\left(1-\frac{x_{2}^{2}}{2 F^{2}}-\frac{y_{2}^{2}}{2 F^{2}}\right)\right]$.

Making use of the assumption that the transverse dimensions of the medium are much larger than the spatial bandwidth of the images and carrying out the integration over the volume of the medium, we can write the electric field at the output plane as

$$
\begin{aligned}
\text { NSR }= & \sum_{m \neq j} \sin ^{2}\left[-t\left(\frac{1}{\lambda_{m}}-\frac{1}{\lambda_{j}}\right) \cos \theta-t\left(\frac{1}{\lambda_{m}}-\frac{1}{\lambda_{j}}\right)\right. \\
& +\frac{t\left(x_{2}^{2}+y_{2}^{2}\right)}{2 F^{2}}\left(\frac{\lambda_{m}}{\lambda_{j}^{2}}-\frac{1}{\lambda_{j}}\right) \\
& -\frac{t \lambda_{m} y_{2}}{F \lambda_{j}}\left(\frac{1}{\lambda_{m}}-\frac{1}{\lambda_{j}}\right) \sin \theta \\
& \left.+\frac{t \lambda_{m}}{2}\left(\frac{1}{\lambda_{m}}-\frac{1}{\lambda_{j}}\right)^{2} \sin ^{2} \theta\right]
\end{aligned}
$$

To minimize the NSR, the spacing between the holograms in frequency (or wavelength) is chosen to place the center pixel of each hologram at the zero of the sinc function of the two adjacent holograms: $\Delta \nu=c /[t(1+\cos \theta)] \equiv \Delta$. To calculate NSR with this frequency schedule, we assume that the individual frequencies are given in terms of $\nu_{m}=m \Delta+\nu_{0}$. Rewriting Eq. (7) in terms of frequency and substituting in the expression for the individual frequencies results in

$$
\begin{aligned}
\mathrm{NSR}= & \sum_{m \neq j} \sin ^{2}[-(m-j) \\
& -\frac{\left(x_{2}^{2}+{y_{2}}^{2}\right)\left(j \Delta+\nu_{0}\right)}{2 F^{2}(1+\cos \theta)\left(m \Delta+\nu_{0}\right)}(m-j) \\
& -\left(\frac{j \Delta+v_{0}}{m \Delta+\nu_{0}}\right) \frac{y_{2}(m-j)}{F(1+\cos \theta)} \sin \theta \\
& \left.+\frac{c(m-j)^{2}}{2 t\left(m \Delta+\nu_{0}\right)(1+\cos \theta)^{2}} \sin ^{2} \theta\right] .
\end{aligned}
$$

The NSR is a function of the hologram number $j$ (or equivalently the reconstructing $\lambda_{j}$ ) and the location

$$
\begin{aligned}
E\left(x_{2}, y_{2}\right) \approx & \sum_{m=-M}^{M} f\left(\frac{-\lambda_{m}}{\lambda_{j}} x_{2}-\frac{F \lambda_{m}}{2 \pi} \Delta K_{m j x}, \frac{-\lambda_{m}}{\lambda_{j}} y_{2}-\frac{F \lambda_{m}}{2 \pi} \Delta K_{m j y}\right) \operatorname{sinc}\left\{\frac { t } { 2 \pi } \left[\Delta K_{m j z}+\left(\frac{2 \pi}{\lambda_{j}}-\frac{2 \pi}{\lambda_{m}}\right)\right.\right. \\
& \left.\left.+\frac{\pi\left(x_{2}{ }^{2}+y_{2}{ }^{2}\right)}{F^{2}}\left(\frac{\lambda_{m}}{\lambda_{j}{ }^{2}}-\frac{1}{\lambda_{j}}\right)+\frac{\lambda_{m}}{\lambda_{j} F}\left(\Delta K_{m j x} x_{2}+\Delta K_{m y j} y_{2}\right)+\frac{\lambda_{m}}{4 \pi}\left(\Delta K_{m j x}{ }^{2}+\Delta K_{m j y}{ }^{2}\right)\right]\right\},
\end{aligned}
$$

where $\Delta K_{m j}=k_{m}-k_{j}$ is the difference between the $m$ th reference wave vector $k_{m}$ and the illuminating beam's wave vector $k_{j}$ and $\Delta K_{m j \alpha}$ is the component of $\Delta K_{m j}$ in the $\alpha$ direction. In relation (6), $t$ is the thickness of the material in the $z$ direction. Note that, when $k_{m}=k_{j}, E_{j}\left(x_{2}, y_{2}\right)=f_{j}\left(-x_{2},-y_{2}\right)$, which is the mirror image of the desired stored image.

The cross-talk noise arises from the $m \neq j$ terms in relation (6). We first write explicit expressions for the Bragg-mismatch terms $\Delta K_{m j x}=0, \Delta K_{m j y}=$ $-\left(2 \pi / \lambda_{m}-2 \pi / \lambda_{j}\right) \sin \theta$, and $\Delta K_{m j z}=-\left(2 \pi / \lambda_{m}-\right.$ $\left.2 \pi / \lambda_{j}\right) \cos \theta$, where $\theta$ is the angle between the reference beam and the optical axis of the output plane. To estimate the noise-to-signal ratio (NSR), we divide the total average noise power by the magnitude squared of the signal. We assume that each pixel of the stored images is an independent random variable, taking the values 0 and 1 with equal probability. In this case, at the output plane $\left(x_{2}, y_{2}\right)$. Given $t, \nu_{0}, F, \theta$, and $N$, the above expression can be numerically evaluated. The NSR on the output plane for the hologram stored at the center frequency $(j=0)$ was calculated by numerically evaluating Eq. (8) with $F=30 \mathrm{~cm}$, $t=1 \mathrm{~cm}, x_{2 \max }=y_{2 \max }=1.5 \mathrm{~cm}, \nu_{0}=6 \times 10^{14} \mathrm{~Hz}$, and $N=401$ holograms. Figure 2 shows the output

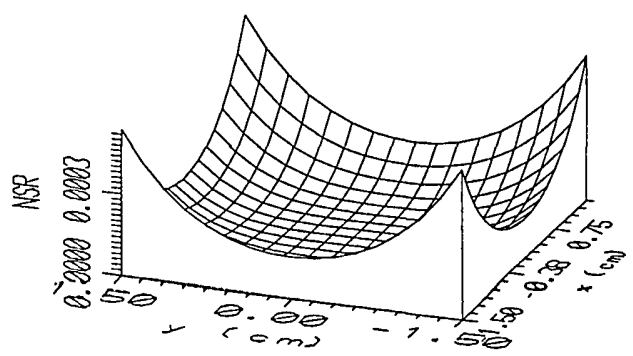

Fig. 2. NSR at the output plane for the $j=0$ hologram with $\theta=0.0^{\circ}$. 


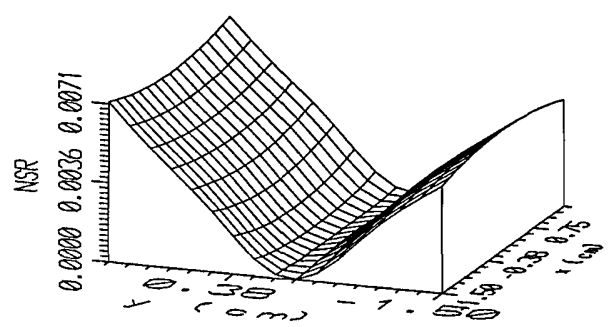

Fig. 3. NSR at the output plane for the $j=0$ hologram with $\theta=15.0^{\circ}$.

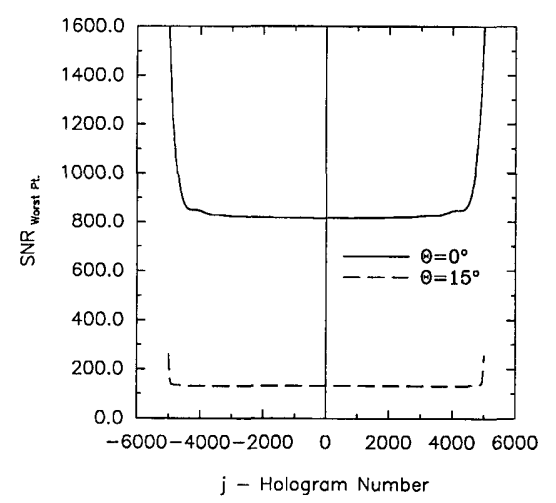

Fig. 4. SNR versus hologram number ( $j$ ) for $N=10,001$ holograms.

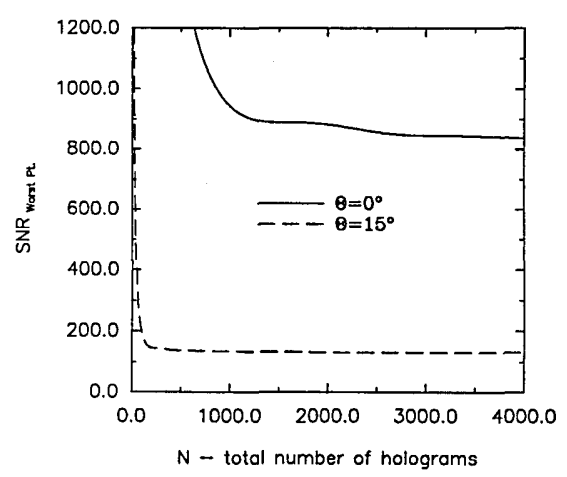

Fig. 5. SNR versus the total number of holograms.

plane for $\theta=0.0^{\circ}$, and Fig. 3 shows the output plane for $\theta=15.0^{\circ}$. In both cases, the maximum NSR is at the edges of the output plane. Setting $x_{2}$ and $y_{2}$ to their maximum values, we can plot the worst-case SNR versus hologram number $j$. Figure 4 shows the results for $\theta=0.0^{\circ}$ and $\theta=15.0^{\circ}$ for $N=10,001$ holograms. Note that the last $j= \pm 5000$ holograms for both cases have SNR's that are twice as large as the middle $(j=0)$ hologram. This is because the $j= \pm M$ holograms have holograms only on one side. Therefore the minimum SNR hologram is always the middle hologram in the frequency schedule. However, even this worst-case SNR is $>800$ for $\theta=0.0^{\circ}$, which is excellent.

From Fig. 4 we know that the middle $(j=0)$ hologram has the minimum SNR and that the noise is symmetric around this hologram. Therefore we can set $j=0$ in Eq. (8) and use it to find a closed-form solution for the maximum NSR. Let us first define $a$ as

$$
a=\frac{\left(x_{2}^{2}+y_{2}^{2}\right)}{2 F^{2}(1+\cos \theta)}+\frac{y_{2}}{F(1+\cos \theta)} \sin \theta .
$$

We can drop the last term in the argument of the sinc function in Eq. (8) because it becomes comparable with the other terms only when $m$ is large. When $m$ is large, the sinc function itself adds a negligible contribution to the summation in Eq. (8). Assuming that $\nu_{0} /\left(m \Delta+\nu_{0}\right) \approx 1$ and using the identities $\sin (x+$ $y)=\sin x \cos y+\cos x \sin y$ and $\sin ^{2} x=1 / 2$ $1 / 2 \cos 2 x$, one can write Eq. (8) as

$$
\mathrm{NSR}_{\max }=\frac{1}{\pi^{2}(1+a)^{2}}\left[\sum_{m=1}^{M} \frac{1}{m^{2}}-\sum_{m=1}^{M} \frac{\cos (2 \pi a m)}{m^{2}}\right] \text {. }
$$

This can be further simplified, if $a \ll 1$ (true for typical lens systems), into

$$
\mathrm{NSR}_{\text {max }} \approx a \approx \frac{\left(x_{2}^{2}+y_{2}^{2}\right)}{2 F^{2}(1+\cos \theta)}+\frac{y_{2}}{F(1+\cos \theta)} \sin \theta,
$$

as the number of holograms stored becomes very large. Note that the best SNR is achieved in the $\theta=0^{\circ}$ geometry. In this case the SNR becomes

$$
\mathrm{SNR}_{\min } \approx \frac{4 F^{2}}{\left(x_{2}^{2}+y_{2}^{2}\right)} \text {. }
$$

Figure 5 is a plot of the SNR at the worst point in the output plane $\left(x_{2}=y_{2}=1.5 \mathrm{~cm}\right)$ versus the total number of holograms stored with the same parameters as given for Figs. 2 and 3. Relation (11) predicts that the SNR goes to 800 at $\theta=0^{\circ}$ and $\sim 127$ at $\theta=15.0^{\circ}$. The plots show that, at $N=4000$ holograms, the SNR is 837 and 131, respectively, showing that the asymptotic expression that we derived is in good agreement with the actual values for typical parameters.

We thank the Defense Advanced Research Projects Agency and Northrop Corporation for support and Geoffrey Burr for helpful discussions.

\section{References}

1. H. Kogelnik, Bell Syst. Tech. J. 48, 2909 (1969).

2. J. J. Amodei and D. L. Staebler, RCA Rev. 33, 71 (1972).

3. F. H. Mok, M. C. Tackitt, and H. M. Stoll, Opt. Lett. 16, 605 (1991).

4. F. T. S. Yu, S. Wu, A. W. Mayers, and S. Rajan, Opt. Commun. 81, 343 (1991).

5. G. A. Rakuljic, V. Leyva, and A. Yariv, Opt. Lett. 17, 1471 (1992).

6. C. Gu, J. Hong, I. McMichael, R. Saxena, and F. H. Mok, J. Opt. Soc. Am. A 9, 1978 (1992).

7. C. Gu and J. Hong, in Annual Meeting, Vol. 23 of 1992 OSA Technical Digest Series (Optical Society of America, Washington, D.C., 1992), paper WT2, p. 111.

8. J. W. Goodman, Introduction to Fourier Optics (McGraw-Hill, New York, 1968), p. 57.

9. J. D. Jackson, Classical Electrodynamics (Wiley, New York, 1975), p. 427.

10. I. S. Gradshteyn and I. M. Ryzhik, Table of Integrals, Series, and Products (Academic, London, 1980), p. 39.

11. M. Abramowitz and I. E. Stegun, Handbook of Mathematical Functions With Formulas, Graphs, and Mathematical Tables (National Bureau of Standards, Washington, D.C., 1972), p. 807. 